\title{
Fuzzy upper and lower generalized $e$-continuous and irresoluteness in double fuzzy topological spaces
}

\author{
P. Periyasamy ${ }^{1}$, V. Chandrasekar ${ }^{2}$, G. Saravanakumar ${ }^{3}$ and A. Vadivel ${ }^{4^{*}}$
}

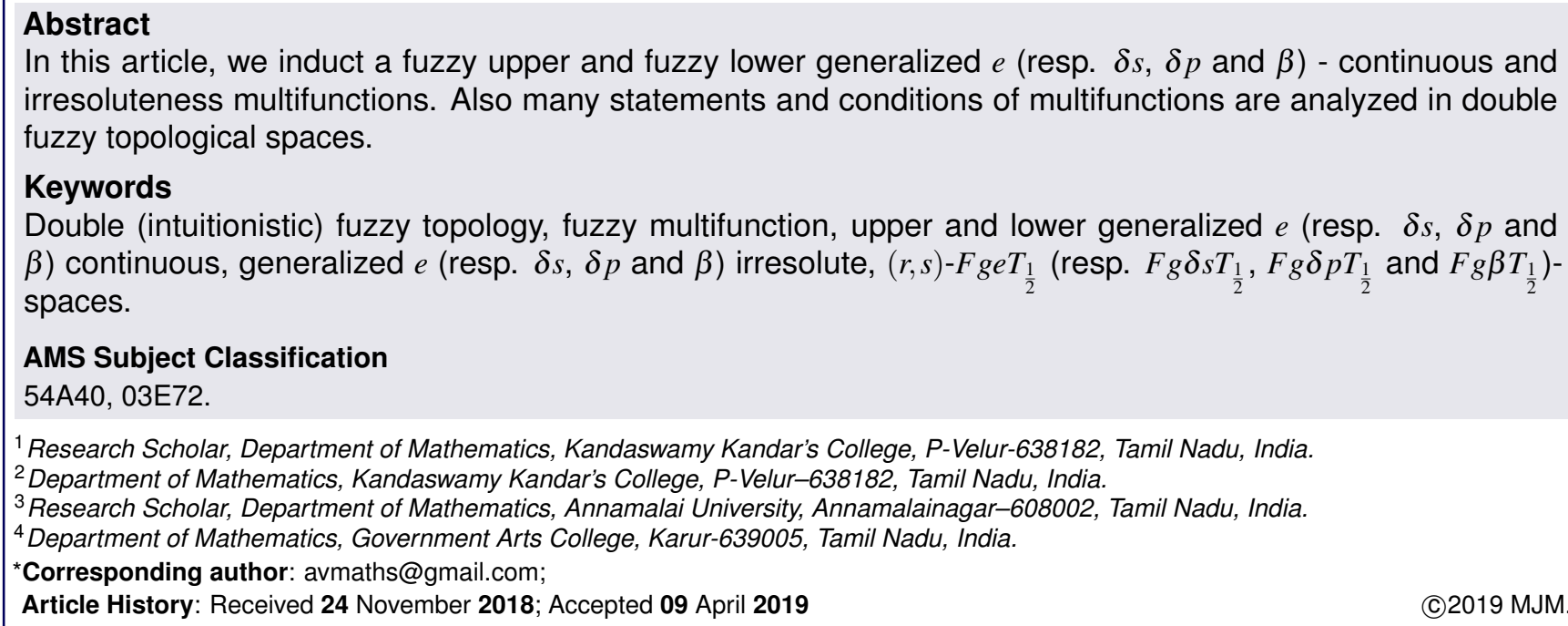

${ }^{1}$ Research Scholar, Department of Mathematics, Kandaswamy Kandar's College, P-Velur-638182, Tamil Nadu, India.

${ }^{2}$ Department of Mathematics, Kandaswamy Kandar's College, P-Velur-638182, Tamil Nadu, India.

${ }^{3}$ Research Scholar, Department of Mathematics, Annamalai University, Annamalainagar-608002, Tamil Nadu, India.

${ }^{4}$ Department of Mathematics, Government Arts College, Karur-639005, Tamil Nadu, India.

*Corresponding author: avmaths@gmail.com;

Article History: Received 24 November 2018; Accepted 09 April 2019

(C)2019 MJM

\section{Contents}

1 Introduction $\ldots \ldots \ldots \ldots \ldots \ldots \ldots \ldots \ldots \ldots \ldots \ldots, 408$

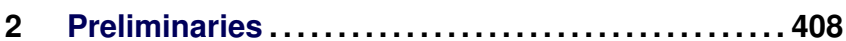

3 Fuzzy upper and lower generalized e (resp. $\delta \mathbf{s}, \delta \mathbf{p}$ and $\beta$ )-continuous and irresolute multifunctions.410

References 413

\section{Introduction}

Chang [10] in 1968 defined fuzzy topological space as every fuzzy set may be open or not. Goguen [15] developed the concept of Chang and redefines the general lattice $L$ in $[0,1]$. Kubiak [20] reprsent topology is itself fuzzy in addition to fuzzy set and Sostak [33] also represent the same. The common approach towards the topological system with power sets was unified in [17]-[21]is the open research field for researchers Now.

Atanassov [6]-[8] introduced Intuitionistic fuzzy set a generalization to fuzzy sets. Intuitionistic fuzzy topology was defined by Coker and his team [11]-[13] in Chang sense. Intuitionistic fuzzy gradation of openness was attributed by Kubiak an d sense [31, 32], in 2005, Gutierrez Garcia and
Rodabaugh [14] after clearing the doubt's of fuzzy intuitionistic they worked under "double".

Concept of multimapping was coined by Berge [9] in topological space also Popa [29, 30] identified the concept of irresolute multimapping. Many authors [4, 5] [25]-[27]) all worked in continuity of multifunction and in concept of fuzzy topology [10]. At present Kerre et al.,[34, 35] identified the continuity of fuzzy multivalued mapping.

All over the paper, nonempty sets will be represent as $X, Y$ etc., $L=[0,1]$ and $L_{0}=(0,1]$. The group of all fuzy sets of $X$ is represented by $L^{X}$. For $\alpha \in L, \underline{\alpha}(x)=\alpha$ for all $x \in X$. A fuzzy point $x_{t}$ for $t \in L_{0}$ is an element of $L^{X}$ such that $x_{t}(y)=\left\{\begin{array}{l}t, \text { if } y=x \\ 0, \text { if } y \neq x .\end{array} \quad\right.$ The group of all fuzzy points in $X$ is represented by $\operatorname{Pt}(X)$. A fuzzy point $x_{t} \in \lambda$ iff $t<\lambda(x)$. All other symbols are as in standard of fuzzy set theory.

\section{Preliminaries}

Definition 2.1. [3] Let $F: X \multimap Y$. Then $F$ is called a fuzzy multifunction ( $F M$, for short) iff $F(x) \in L^{Y}$ for each $x \in X$. The degree of membership of $y$ in $F(x)$ is denoted by $F(x)(y)=G_{F}(x, y)$ for any $(x, y) \in X \times Y$. 
The domain of $F$, denoted by $\operatorname{dom}(F)$ and the range of $F$, denoted by $\operatorname{rng}(F)$ for any $x \in X, y \in Y: \operatorname{dom}(F)=\bigvee_{y \in Y} G_{F}(x, y)$ and $\operatorname{rng}(F)(y)=\vee_{x \in X} G_{F}(x, y)$.

Definition 2.2. [3] Let $F: X \multimap Y$ be a FM. Then $F$ is called normalized iff for each $x \in X$, there exists $y_{0} \in Y$ such that $G_{F}\left(x, y_{0}\right)=1$.

Definition 2.3. [3] Let $F: X \multimap Y$ be a FM. Then

(i) The image of $\lambda \in L^{X}$ is a fuzzy set $F(\lambda) \in L^{Y}$ and defined by: $F(\lambda)(y)=\vee_{x \in X}\left[G_{F}(x, y) \wedge \lambda(x)\right]$.

(ii) The lower inverse of $\mu \in L^{Y}$ is a fuzzy set $F^{l}(\mu) \in L^{X}$ and defined by: $F^{l}(\mu)(x)=\vee_{y \in Y}\left[G_{F}(x, y) \wedge \mu(y)\right]$.

(iii) The upper inverse of $\mu \in L^{Y}$ is a fuzzy set $F^{u}(\mu) \in L^{X}$ and defined by: $F^{u}(\mu)(x)=\wedge_{y \in Y}\left[G_{F}^{c}(x, y) \vee \mu(y)\right]$.

Theorem 2.4. [3] Let $F: X \multimap Y$ be a FM. Then

(i) $F\left(\lambda_{1}\right) \leq F\left(\lambda_{2}\right)$ if $\lambda_{1} \leq \lambda_{2}$.

(ii) $F^{l}\left(\mu_{1}\right) \leq F^{l}\left(\mu_{2}\right.$ and $F^{u}\left(\mu_{1}\right) \leq F^{u}\left(\mu_{2}\right.$ if $\mu_{1} \leq \mu_{2}$.

(iii) $F^{l}(\underline{1}-\mu)=\underline{1}-F^{u}(\mu)$.

(iv) $F^{u}(\underline{1}-\mu)=\underline{1}-F^{l}(\mu)$.

Definition 2.5. [32, 36] A double fuzzy topology on $X$ is a pair $(\hat{\tau}, \breve{\tau})$ of the mappings $\hat{\tau}, \breve{\tau}: L^{X} \rightarrow L$ which satisfy the following conditions:

(i) $\hat{\tau}(\lambda)+\breve{\tau}(\lambda) \leq 1, \forall \lambda \in L^{X}$

(ii) $\hat{\tau}\left(\lambda_{1} \wedge \lambda_{2}\right) \geq \hat{\tau}\left(\lambda_{1}\right) \wedge \hat{\tau}\left(\lambda_{2}\right)$ and $\breve{\tau}\left(\lambda_{1} \wedge \lambda_{2}\right) \leq \breve{\tau}\left(\lambda_{1}\right) \vee$ $\breve{\tau}\left(\lambda_{2}\right), \forall \lambda_{1}, \lambda_{2} \in L^{X}$.

(iii) $\hat{\tau}\left(\bigvee_{i \in \Gamma} \lambda_{i}\right) \geq \bigwedge_{X \in \Gamma} \tilde{\tau}\left(\lambda_{i}\right)$ and $\breve{\tau}\left(\bigvee_{i \in \Gamma} \lambda_{i}\right) \leq \bigvee_{i \in \Gamma} \breve{\tau}\left(\lambda_{i}\right)$, $\forall\left\{\lambda_{i}\right\}_{i \in \Gamma} \subset L^{X}$.

The triplet $(X, \hat{\tau}, \breve{\tau})$ is called a double (intuitionistic) fuzzy topological space (dfts, for short). $\tilde{\tau}(\lambda)$ and $\breve{\tau}(\lambda)$ may be interpreted as a gradation of openness and a gradation of nonopenness for $\lambda \in L^{X}$, respectively.

Theorem 2.6. [1, 22] Let $(X, \tilde{\tau}, \breve{\tau})$ be a dfts. Then for each $\lambda \in L^{X}, r \in L_{0}$ and $s \in L_{1}$, we define operators $C_{\hat{\tau}, \tilde{\tau}}$ and $I_{\hat{\tau}, \breve{\tau}}: L^{X} \times L_{0} \times L_{1} \rightarrow L^{X}$ as follows: $C_{\hat{\tau}, \breve{\tau}}(\lambda, r, s)=\bigwedge\{\mu \in$ $\left.L^{X}: \lambda \leq \mu, \hat{\tau}(\underline{1}-\mu) \geq r, \breve{\tau}(\underline{1}-\mu) \leq s\right\}$ and $I_{\hat{\tau}, \breve{\tau}}(\lambda, r, s)=\bigvee\left\{\mu \in L^{X}: \mu \leq \lambda . \hat{\tau}(\mu) \geq r, \breve{\tau}(\mu) \leq s\right\}$.

For $\lambda, \mu \in L^{X}$ and $r \in L_{0}, s \in L_{1}$ the opertaor $C_{\hat{\tau}, \breve{\tau}}$ satisfies the following statements:

(i) $C_{\hat{\tau}, \breve{\tau}}(\underline{0}, r, s)=\underline{0}$.

(ii) $\lambda \leq C_{\hat{\tau}, \breve{\tau}}(\lambda, r, s)$.

(iii) $C_{\hat{\tau}, \breve{\tau}}(\lambda, r, s) \vee C_{\hat{\tau}, \breve{\tau}}(\mu, r, s)=C_{\hat{\tau}, \breve{\tau}}(\lambda \vee \mu, r, s)$

(iv) $C_{\hat{\tau}, \breve{\tau}}(\lambda, r, s)=\lambda$ iff $\hat{\tau}(\underline{1}-\lambda) \geq r$ and $\breve{\tau}(\underline{1}-\lambda) \leq s$. (v) $C_{\hat{\tau}, \breve{\tau}}\left(C_{\hat{\tau}, \breve{\tau}}(\lambda, r, s), r, s\right)=C_{\hat{\tau}, \breve{\tau}}(\lambda, r, s)$

(vi) $C_{\hat{\tau}, \breve{\tau}}(\underline{1}-\lambda, r, s)=\underline{1}-I_{\hat{\tau}, \breve{\tau}}(\lambda, r, s)$ and $I_{\hat{\tau}, \breve{\tau}}(\underline{1}-\lambda, r, s)=$ $\underline{1}-C_{\hat{\tau}, \breve{\tau}}(\lambda, r, s)$.

Definition 2.7. [1] Let $(X, \tilde{\tau}, \breve{\tau})$ be a dfts. Then for each $\lambda \in L^{X}, r \in L_{0}$ and $s \in L_{1}$ : a fuzzy set $\lambda$ is called an

(i) $(r, s)$-generalized fuzzy open (briefly, $(r, s)$-gfo) iff $\mu \leq$ $I_{\hat{\tau}, \breve{\tau}}(\lambda, r, s)$ whenever $\mu \leq \lambda$ and $\hat{\tau}(\underline{1}-\mu) \geq r, \quad \breve{\tau}(\underline{1}-$ $\mu) \leq s$.

(ii) $(r, s)$-generalized fuzzy closed (briefly, $(r, s)$-gfc) iff $C_{\hat{\tau}, \breve{\tau}}$ $(\lambda, r, s) \leq \mu$ whenever $\lambda \leq \mu$ and $\hat{\tau}(\mu) \geq r, \breve{\tau}(\mu) \leq s$.

Theorem 2.8. [1] Let $(X, \hat{\tau}, \breve{\tau})$ be a dfts. Then for each $\lambda \in$ $L^{X}, r \in L_{0}$ and $s \in L_{1}$, we have

(i) If $\tilde{\tau}(\underline{1}-\lambda) \geq r, \breve{\tau}(\underline{1}-\lambda) \leq s$, then $\lambda$ is $(r, s)-g f c$.

(ii) If $\lambda$ is $(r, s)-g f c$, then $\lambda$ is $(r, s)-g f s c$.

Theorem 2.9. [1] A dfts $(X, \hat{\tau}, \breve{\tau})$ is said to be: $(r, s)-F T_{\frac{1}{2}}$ iff $\hat{\tau}(\underline{1}-\lambda) \geq r, \breve{\tau}(\underline{1}-\lambda) \leq s$ for each $\lambda \in L^{X}$ is $(r, s)-g f c$.

Definition 2.10. [28] Let $(X, \hat{\tau}, \breve{\tau})$ be a dfts. Then for each $\lambda \in L^{X}, r \in L_{0}$ and $s \in L_{1}$ : a fuzzy set $\lambda$ is called an $(r, s)$ fuzzy regular open (briefly, $(r, s)$-fro) $\lambda=I_{\hat{\tau}, \bar{\tau}} C_{\hat{\tau}, \breve{\tau}}(\lambda)$ and $(r, s)$ fuzzy regular closed (briefly, $(r, s)-f r c)$ if $\lambda=C_{\hat{\tau}, \breve{\tau}} I_{\hat{\tau}, \breve{\tau}}(\lambda)$.

Definition 2.11. [28] Let $(X, \hat{\tau}, \breve{\tau})$ be a dfts. Then for each $\lambda \in L^{X}, r \in L_{0}$ and $s \in L_{1}$, we define operators $\delta C_{\hat{\tau}, \breve{\tau}}$ and $\delta I_{\hat{\tau}, \breve{\tau}}: L^{X} \times L_{0} \times L_{1} \rightarrow L^{X}$ as follows: $\delta C_{\hat{\tau}, \breve{\tau}}(\lambda, r, s)=\bigwedge\{\mu \in$ $L^{X}: \lambda \leq \mu, \mu$ is $\left.(r, s)-f r c\right\}$ and $\delta I_{\hat{\tau}, \breve{\tau}}(\lambda, r, s)=\bigvee\left\{\mu \in L^{X}\right.$ : $\mu \leq \lambda, \mu$ is $(r, s)-$ fro\}.

Definition 2.12. [28] Let $(X, \hat{\tau}, \breve{\tau})$ be a dfts. Then for each $\lambda \in L^{X}, r \in L_{0}$ and $s \in L_{1}$ : a fuzzy set $\lambda$ is called an

(i) $(r, s)$-fuzzy $\delta$ semi open (briefly, $(r, s)-f \delta$ so) if $\lambda \leq$ $C_{\hat{\tau}, \breve{\tau}}\left(\delta I_{\hat{\tau}, \breve{\tau}}(\lambda, r, s), r, s\right)$ and $(r, s)$-fuzzy $\delta$ semi closed (briefly, $(r, s)-f \delta s c)$ if $\lambda \geq I_{\hat{\tau}, \tilde{\tau}}\left(\delta C_{\hat{\tau}, \breve{\tau}}(\lambda, r, s), r, s\right)$.

(ii) $(r, s)$-fuzzy $\delta$ pre open (briefly, $(r, s)$ - $f \delta p o)$ if $\lambda \leq I_{\hat{\tau}, \breve{\tau}}($ $\left.\delta C_{\hat{\tau}, \breve{\tau}}(\lambda, r, s), r, s\right)$ and $(r, s)$-fuzzy $\delta$ pre closed (briefly, $(r, s)-f \delta p c)$ if $\lambda \geq C_{\hat{\tau}, \breve{\tau}}\left(\delta I_{\hat{\tau}, \breve{\tau}}(\lambda, r, s), r, s\right)$.

(iii) $(r, s)$-fuzzy $\beta$ open (briefly, $(r, s)-f \beta o)$ if $\lambda \leq C_{\hat{\tau}, \breve{\tau}}$ $\left(I_{\hat{\tau}, \breve{\tau}}\left(C_{\hat{\tau}, \breve{\tau}}(\lambda, r, s), r, s\right), r, s\right)$ and $(r, s)$-fuzzy $\beta$ closed (briefly, $(r, s)-f \beta c)$ if $\lambda \geq I_{\hat{\tau}, \breve{\tau}}\left(C_{\hat{\tau}, \breve{\tau}}\left(I_{\hat{\tau}, \breve{\tau}}(\lambda, r, s), r, s\right)\right.$, $r, s)$.

(iv) $(r, s)$-fuzzy e-open (briefly, $(r, s)$-feo) if $\lambda \leq C_{\hat{\tau}, \breve{\tau}}$ $\left(\delta I_{\hat{\tau}, \breve{\tau}}(\lambda, r, s), r, s\right) \vee I_{\hat{\tau}, \breve{\tau}}\left(\delta C_{\hat{\tau}, \breve{\tau}}((\lambda, r, s), r, s)\right.$ and $(r, s)$ -fuzzy e-closed (briefly, $(r, s)$-fec) if $\lambda \geq I_{\hat{\tau}, \breve{\tau}}\left(\delta C_{\hat{\tau}, \breve{\tau}}\right.$ $(\lambda, r, s), r, s) \wedge C_{\hat{\tau}, \breve{\tau}}\left(\delta I_{\hat{\tau}, \breve{\tau}}((\lambda, r, s), r, s)\right.$.

Definition 2.13. [28] Let $(X, \hat{\tau}, \breve{\tau})$ be a dfts. Then for each $\lambda \in L^{X}, r \in L_{0}$ and $s \in L_{1}$, we define operators $e C_{\hat{\tau}, \breve{\tau}}$ (resp. $\delta S C_{\hat{\tau}, \breve{\tau}}, \delta P C_{\hat{\tau}, \breve{\tau}}$ and $\beta C_{\hat{\tau}, \breve{\tau}}$ ) and $e I_{\hat{\tau}, \breve{\tau}}$ (resp. $\delta S I_{\hat{\tau}, \breve{\tau}}, \delta P I_{\hat{\tau}, \breve{\tau}}$ and

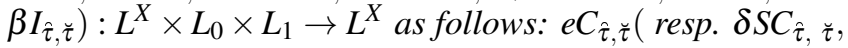


$\delta P C_{\hat{\tau}, \breve{\tau}}$ and $\left.\beta C_{\hat{\tau}, \breve{\tau}}\right)(\lambda, r, s)=\bigwedge\left\{\mu \in L^{X}: \lambda \leq \mu, \mu\right.$ is $(r, s)-$ fec (resp. $f \delta s c, f \delta p c$ and $f \beta c)\}$.

$e I_{\hat{\tau}, \breve{\tau}}\left(\operatorname{resp} . \quad \delta S I_{\hat{\tau}, \breve{\tau}}, \delta P I_{\hat{\tau}, \breve{\tau}}\right.$ and $\left.\beta I_{\hat{\tau}, \breve{\tau}}\right)(\lambda, r, s)=\vee\left\{\mu \in L^{X}:\right.$ $\mu \leq \lambda, \mu$ is $(r, s)-$ feo (resp. $f \delta$ so, $f \delta$ po and $f \beta o)\}$.

For $\lambda, \mu \in L^{X}$ and $r \in L_{0}, s \in L_{1}$ the operator $e C_{\hat{\tau}, \breve{\tau}}$ satisfies the following conditions:

(i) $e C_{\hat{\tau}, \breve{\tau}}(\underline{0}, r, s)=\underline{0}$.

(ii) $\lambda \leq e C_{\hat{\tau}, \breve{\tau}}(\lambda, r, s)$.

(iii) $e C_{\hat{\tau}, \breve{\tau}}(\lambda, r, s) \vee e C_{\hat{\tau}, \breve{\tau}}(\mu, r, s)=e C_{\hat{\tau}, \breve{\tau}}(\lambda \vee \mu, r, s)$

(iv) $e C_{\hat{\tau}, \breve{\tau}}(\lambda, r, s)=\lambda$ iff $\mu$ is $(r, s)$-fec.

(v) $e C_{\hat{\tau}, \breve{\tau}}\left(e C_{\hat{\tau}, \breve{\tau}}(\lambda, r, s), r, s\right)=e C_{\hat{\tau}, \breve{\tau}}(\lambda, r, s)$.

(vi) $e C_{\hat{\tau}, \breve{\tau}}(\underline{1}-\lambda, r, s)=\underline{1}-e I_{\hat{\tau}, \breve{\tau}}(\lambda, r, s)$ and $e I_{\hat{\tau}, \breve{\tau}}(\underline{1}-\lambda, r, s)$ $=\underline{1}-e C_{\hat{\tau}, \breve{\tau}}(\lambda, r, s)$.

Similarly the other operators (i.e) $\delta S C_{\hat{\tau}, \breve{\tau}}, \delta P C_{\hat{\tau}, \breve{\tau}}$ and $\beta C_{\hat{\tau}, \breve{\tau}}$ satisfies the above conditions.

Definition 2.14. [28] Let $(X, \hat{\tau}, \breve{\tau})$ be a dfts. Then for each $\lambda \in L^{X}, r \in L_{0}$ and $s \in L_{1}:$ a fuzzy set $\lambda$ is called

(i) $(r, s)$ generalized fuzzy $\delta$ semi open (briefly, $(r, s)$-gf $\delta$ so) if $\mu \leq \delta S I_{\hat{\tau}, \breve{\tau}}(\lambda, r, s)$ whenever $\mu \leq \lambda$ and $\hat{\tau}(\underline{1}-\mu) \geq$ $r, \breve{\tau}(\underline{1}-\mu) \leq s$.

(ii) $(r, s)$-generalized fuzzy $\delta$ pre open (briefly, $(r, s)$-gf $\delta$ po) if $\mu \leq \delta P I_{\hat{\tau}, \breve{\tau}}(\lambda, r, s)$ whenever $\mu \leq \lambda$ and $\hat{\tau}(\underline{1}-\mu) \geq$ $r, \breve{\tau}(\underline{1}-\mu) \leq s$.

(iii) $(r, s)$-generalized fuzzy $\beta$ open (briefly, $(r, s)$-gf $\beta o$ ) if $\mu \leq \beta I_{\hat{\tau}, \breve{\tau}}(\lambda, r, s)$ whenever $\mu \leq \lambda$ and $\hat{\tau}(\underline{1}-\mu) \geq$ $r, \breve{\tau}(\underline{1}-\mu) \leq s$.

(iv) $(r, s)$-generalized fuzzy e open (briefly, $(r, s)$-gfeo) if $\mu \leq e I_{\hat{\tau}, \breve{\tau}}(\lambda, r, s)$ whenever $\mu \leq \lambda$ and $\hat{\tau}(\underline{1}-\mu) \geq r$, $\breve{\tau}(\underline{1}-\mu) \leq s$.

(v) $(r, s)$-generalized fuzzy $\delta$ semi closed (briefly, $(r, s)$ gf $\delta s c)$ if $\delta S C_{\hat{\tau}, \breve{\tau}}(\lambda, r, s) \leq \mu$ whenever $\lambda \leq \mu$ and $\hat{\tau}(\mu) \geq r, \breve{\tau}(\mu) \leq s$.

(vi) $(r, s)$ generalized fuzzy $\delta$ pre closed (briefly, $(r, s)-g f \delta p c)$ if $\delta P C_{\hat{\tau}, \breve{\tau}}(\lambda, r, s) \leq \mu$ whenever $\lambda \leq \mu$ and $\hat{\tau}(\mu) \geq$ $r, \breve{\tau}(\mu) \leq s$.

(vii) $(r, s)$-generalized fuzzy $\beta$ closed (briefly, $(r, s)-g f \beta c$ ) if $\beta C_{\hat{\tau}, \breve{\tau}}(\lambda, r, s) \leq \mu$ whenever $\lambda \leq \mu$ and $\hat{\tau}(\mu) \geq r$, $\breve{\tau}(\mu) \leq s$.

(viii) $(r, s)$-generalized fuzzy e closed (briefly, $(r, s)$-gfec) if $e C_{\hat{\tau}, \breve{\tau}}(\lambda, r, s) \leq \mu$ whenever $\lambda \leq \mu$ and $\tilde{\tau}(\mu) \geq r, \breve{\tau}(\mu) \leq$ $s$.

Definition 2.15. [16] Let $F: X \multimap Y$ be a FM between two dfts's $(X, \hat{\tau}, \breve{\tau}),(Y, \hat{\eta}, \breve{\eta})$ and $r \in L_{0}, s \in L_{1}$. Then $F$ is called: (i) Fuzzy upper generalized continuous (FUGcontinuous, for short) at a fuzzy point $x_{t} \in \operatorname{dom}(F)$ iff $x_{t} \in F^{u}(\mu)$ for each $\mu \in L^{Y}$ and $\hat{\eta}(\mu) \geq r, \breve{\eta}(\mu) \leq s$ there exists $\lambda \in L^{X}, \lambda$ is $(r, s)$-gfo and $x_{t} \in \lambda$ such that $\lambda \wedge \operatorname{dom}(F) \leq F^{u}(\mu) . F$ is $F U G$-continuous iff it is $F U G$-continuous at every $x_{t} \in \operatorname{dom}(F)$.

(ii) Fuzzy lower generalized continuous (FLGcontinuous, for short) at a fuzzy point $x_{t} \in \operatorname{dom}(F)$ iff $x_{t} \in F^{l}(\mu)$ for each $\mu \in L^{Y}$ and $\hat{\eta}(\mu) \geq r, \breve{\eta}(\mu) \leq s$ there exists $\lambda \in L^{X}, \lambda$ is $(r, s)$-gfo and $x_{t} \in \lambda$ such that $\lambda \wedge \operatorname{dom}(F) \leq F^{l}(\mu) . \quad F$ is FLG-continuous iff it is FLG-continuous at every $x_{t} \in \operatorname{dom}(F)$.

Proposition 2.16. [16] Let $F: X \multimap Y$ be a FM between two dfts's $(X, \hat{\tau}, \breve{\tau}),(Y, \hat{\eta}, \breve{\eta})$ and $r \in L_{0}, s \in L_{1}$. If $F$ is normalized, then $F$ is:

(i) FUG-continuous at $x_{t} \in \operatorname{dom}(F)$ iff $x_{t} \in F^{u}(\mu) \forall \mu \in$ $L^{Y}$ and $\hat{\eta}(\mu) \geq r, \breve{\eta}(\mu) \leq s$ there exists $\lambda \in L^{X}, \lambda$ is $(r, s)$-gfo and $x_{t} \in \lambda$ such that $\lambda \leq F^{u}(\mu)$.

(ii) FUG-irresolute at $x_{t} \in \operatorname{dom}(F)$ iff $x_{t} \in F^{u}(\mu) \forall \mu \in L^{Y}$ and $\hat{\eta}(\mu) \geq r, \breve{\eta}(\mu) \leq s$ there exists $\lambda \in L^{X}, \lambda$ is $(r, s)$ gfo and $x_{t} \in \lambda$ such that $\lambda \leq F^{u}(\mu)$.

\section{Fuzzy upper and lower generalized e (resp. $\delta \mathbf{s}, \delta$ p and $\beta$ )-continuous and irresolute multifunctions}

Definition 3.1. Let $F: X \multimap Y$ be a FM between two dfts's $(X, \hat{\tau}, \breve{\tau}),(Y, \hat{\eta}, \breve{\eta})$ and $r \in L_{0}, s \in L_{1}$. Then $F$ is called:

(i) Fuzzy upper generalized e (resp. $\delta S, \delta P$ and $\beta$ ) (FUGe, $F U G \delta S, F U G \delta P$ and $F U G \beta$, for short) continuous at a fuzzy point $x_{t} \in \operatorname{dom}(F)$ iff $x_{t} \in F^{u}(\mu)$ for each $\mu \in L^{Y}$ and $\hat{\eta}(\mu) \geq r, \breve{\eta}(\mu) \leq s$ there exists $\lambda \in L^{X}, \lambda$ is $(r, s)$-gfeo (resp. gf $\delta$ so, gf $\delta$ po and $g f \beta o$ ) and $x_{t} \in$ $\lambda$ such that $\lambda \wedge \operatorname{dom}(F) \leq F^{u}(\mu)$. F is FUGe (resp. FUGSS, FUGSP and FUG $\beta$ )-continuous iff it is FUGe

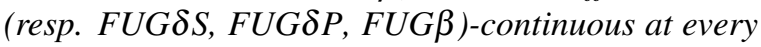
$x_{t} \in \operatorname{dom}(F)$.

(ii) Fuzzy lower generalized e (resp. $\delta S, \delta P$ and $\beta$ ) (FLGe, $F L G \delta S, F L G \delta P$ and FLG $\beta$, for short) continuous at a fuzzy point $x_{t} \in \operatorname{dom}(F)$ iff $x_{t} \in F^{u}(\mu)$ for each $\mu \in L^{Y}$ and $\hat{\eta}(\mu) \geq r, \breve{\eta}(\mu) \leq s$ there exists $\lambda \in L^{X}, \lambda$ is $(r, s)$ -

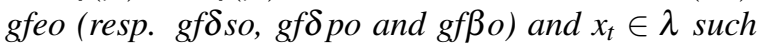
that $\lambda \leq F^{l}(\mu)$. F is FLGe (resp. FLG $\delta S, F L G \delta P$ and FLG $\beta$ )-continuous iff it is FLGe (resp. FLG $\delta S$, $F L G \delta P$ and $F L G \beta)$-continuous at every $x_{t} \in \operatorname{dom}(F)$.

Definition 3.2. Let $F: X \multimap Y$ be a FM between two dfts's $(X, \hat{\tau}, \breve{\tau}),(Y, \hat{\eta}, \breve{\eta})$ and $r \in L_{0}, s \in L_{1}$. Then $F$ is called:

(i) Fuzzy upper generalized e (resp. $\delta S, \delta P$ and $\beta$ ) (FUGe, $F U G \delta S, F U G \delta P$ and $F U G \beta$, for short) irresolute at a fuzzy point $x_{t} \in \operatorname{dom}(F)$ iff $x_{t} \in F^{u}(\mu)$ for each $\mu \in L^{Y}$ 
and $\mu$ is $(r, s)$-gfeo (resp. $g f \delta$ so, $g f \delta$ po and $g f \beta o$ ) there exists $\lambda \in L^{X}, \lambda$ is (r,s)-gfeo (resp. gf $\delta$ so, gf $\delta$ po and $g f \beta o$ ) and $x_{t} \in \lambda$ such that $\lambda \wedge \operatorname{dom}(F) \leq F^{u}(\mu) . F$

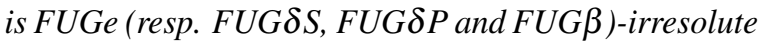

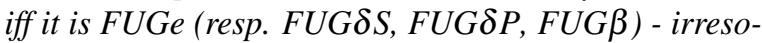
lute at every $x_{t} \in \operatorname{dom}(F)$.

(ii) Fuzzy lower generalized e (resp. $\delta S, \delta P, \beta)(F U G e$, $F U G \delta S, F U G \delta P$ and $F U G \beta$, for short) irresolute at a fuzzy point $x_{t} \in \operatorname{dom}(F)$ iff $x_{t} \in F^{u}(\mu)$ for each $\mu \in L^{Y}$ and $\mu$ is $(r, s)$-gfeo (resp. $g f \delta$ so, $g f \delta p o$ and $g f \beta o$ ) there exists $\lambda \in L^{X}, \lambda$ is (r,s)-gfeo (resp. gf $\delta$ so, gf $\delta$ po and $g f \beta o$ ) and $x_{t} \in \lambda$ such that $\lambda \leq F^{l}(\mu)$. F is FLGe

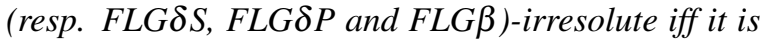

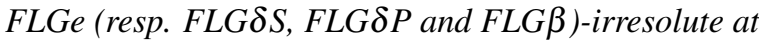
every $x_{t} \in \operatorname{dom}(F)$.

Proposition 3.3. Let $F: X \multimap Y$ be a FM between two dfts's $(X, \hat{\tau}, \breve{\tau}),(Y, \hat{\eta}, \breve{\eta})$ and $r \in L_{0}, s \in L_{1}$. Then $F$ is normalized, and $F$ is:

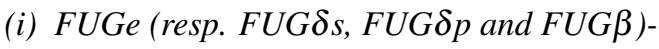
continuous at $x_{t} \in \operatorname{dom}(F)$ iff $x_{t} \in F^{u}(\mu) \forall \mu \in L^{Y}$ and $\hat{\eta}(\mu) \geq r, \breve{\eta}(\mu) \leq$ s there exists $\lambda \in L^{X}, \lambda$ is $(r, s)$-gfeo

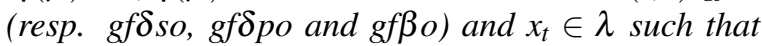
$\lambda \leq F^{u}(\mu)$.

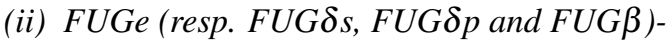
irresolute at $x_{t} \in \operatorname{dom}(F)$ iff $x_{t} \in F^{u}(\mu) \forall \mu \in L^{Y}$ and $\mu$ is $(r, s)$-gfeo (resp. gf $\delta$ so, gf $\delta p o$ and $g f \beta o$ ) there exists $\lambda \in L^{X}, \lambda$ is (r,s)-gfeo (resp. gf $\delta$ so, gf $\delta$ po and gf $\beta$ o $)$ and $x_{t} \in \lambda$ such that $\lambda \leq F^{u}(\mu)$.

The following implications hold:

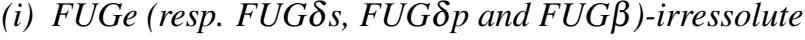

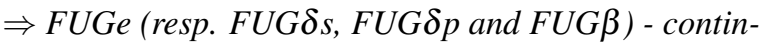
uous.

(ii) $F L G e($ resp. $F L G \delta s, F L G \delta p$ and $F L G \beta)$-irressolute $\Rightarrow$

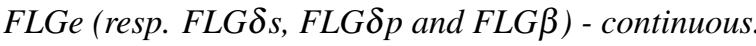

In general the converses are not true.

Theorem 3.4. Let $F: X \multimap Y$ be a FM between two dfts's $(X, \tilde{\tau}, \breve{\tau}),(Y, \hat{\eta}, \breve{\eta})$ and $\mu \in L^{Y} \quad r \in L_{0}, s \in L_{1}$. Suppose that one of the following properties hold:

(i) $F^{u}(\mu)$ is $(r, s)$-gfeo (resp. $(r, s)$-gf $\delta$ so, $(r, s)$-gf $\delta$ po and $(r, s)$-gf $\beta o$ ), for any $\hat{\eta}(\mu) \geq r, \breve{\eta}(\mu) \leq s$.

(ii) $F^{l}(\mu)$ is $(r, s)$-gfec (resp. $(r, s)$-gf $\delta s c,(r, s)$-gf $\delta p c$ and $(r, s)$-gf $\beta c)$, for any $\hat{\eta}(\underline{1}-\mu) \geq r, \breve{\eta}(\underline{1}-\mu) \leq s$.

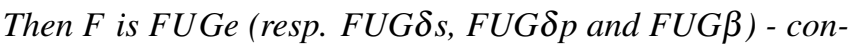
tinuous.

Proof. (i) $\Rightarrow$ (ii): Let $\mu \in L^{Y}$ and $\hat{\eta}(\underline{1}-\mu) \geq r, \breve{\eta}(1-\mu) \leq s$ hence by (i) $F^{u}(\underline{1}-\mu)=\underline{1}-F^{l}(\mu)$ is $(r, s)$-gfec. Then $F^{l}(\mu)$ is $(r, s)$-gfec. (ii) $\Rightarrow$ (i): It is similar to that of (i) $\Rightarrow$ (ii).

Suppose that (i) holds. Let $x_{t} \in \operatorname{dom}(F), \hat{\eta}(\mu) \geq r, \breve{\eta}(\mu) \leq s$ and $x_{t} \in F^{u}(\mu)$ we have by (i), $F^{u}(\mu)=\lambda$ (say) is $(r, s)$ gfeo then, there exits $\lambda$ is $(r, s)$-gfeo and $x_{t} \in \lambda$ such that $\lambda \wedge \operatorname{dom}(F) \leq F^{u}(\mu)$. Thus $F$ is $F U G e$-continuous.

Proof of the other cases are similar.

Theorem 3.5. Let $F: X \multimap Y$ be a FM between two dfts's $(X, \tilde{\tau}, \breve{\tau}),(Y, \hat{\eta}, \breve{\eta})$ and $\mu \in L^{Y} r \in L_{0}, s \in L_{1}$. Suppose that one of the following properties hold:

(i) $F^{u}(\mu)$ is $(r, s)$-gfeo (resp. $(r, s)$-gf $\delta$ so, $(r, s)$-gf $\delta$ po and $(r, s)$-gf $\beta o)$, for any $\mu$ is $(r, s)$-gfeo (resp. $(r, s)$-gf $\delta$ so, $(r, s)$-gf $\delta$ po and $(r, s)-g f \beta o)$.

(ii) $F^{l}(\mu)$ is $(r, s)$-gfec (resp. $(r, s)-g f \delta s c,(r, s)$-gfopc and $(r, s)$-gf $\beta c)$, for any $\mu$ is $(r, s)$-gfec (resp. $(r, s)$-gf $\delta s c$, $(r, s)-g f \delta p c$ and $(r, s)-g f \beta c)$.

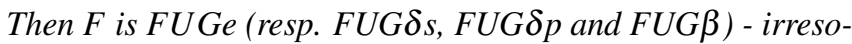
lute.

Proof. (i) $\Rightarrow$ (ii): Let $\mu$ is $(r, s)$-gfec hence by (i) $F^{u}(\underline{1}-\mu)=$ $1-F^{l}(\mu)$ is $(r, s)$-gfeo. Then $F^{l}(\mu)$ is $(r, s)$-gfec.

(ii) $\Rightarrow$ (i): It is similar to that of (i) $\Rightarrow$ (ii).

Suppose that (i) holds. Let $x_{t} \in \operatorname{dom}(F), \mu$ is $(r, s)$-gfeo and $x_{t} \in F^{u}(\mu)$ we have by (i), $F^{u}(\mu)=\lambda$ (say) is $(r, s)$ gfeo then, there exits $\lambda$ is $(r, s)$-gfeo and $x_{t} \in \lambda$ such that $\lambda \wedge \operatorname{dom}(F) \leq F^{u}(\mu)$. Thus $F$ is $F U G e$-irresolute.

Proof of the other cases are similar.

Theorem 3.6. Let $F: X \multimap Y$ be a FM between two dfts's $(X, \hat{\tau}, \breve{\tau}),(Y, \hat{\eta}, \breve{\eta})$ and $\mu \in L^{Y}, r \in L_{0}, s \in L_{1}$. Suppose that one of the following properties hold:

(i) $F^{l}(\mu)$ is $(r, s)$-gfeo (resp. $(r, s)$-gf $\delta$ so, $(r, s)$-gf $\delta$ po and $(r, s)$-gf $\beta o$ ), for any $\hat{\eta}(\mu) \geq r, \breve{\eta}(\mu) \leq s$.

(ii) $F^{u}(\mu)$ is $(r, s)$-gfec (resp. $(r, s)-g f \delta s c,(r, s)$-gf $\delta p c$ and $(r, s)-g f \beta c)$, for any $\hat{\eta}(\underline{1}-\mu) \geq r, \breve{\eta}(\underline{1}-\mu) \leq s$.

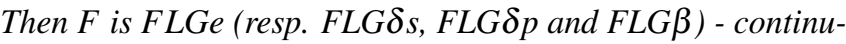
ous.

Proof. This can be proved in similar way as Theorem 3.4

Theorem 3.7. Let $F: X \multimap Y$ be a FM between two dfts's $(X, \hat{\tau}, \breve{\tau}),(Y, \hat{\eta}, \breve{\eta})$ and $\mu \in L^{Y}, r \in L_{0}, s \in L_{1}$. Suppose that one of the following properties hold:

(i) $F^{l}(\mu)$ is $(r, s)$-gfeo (resp. $(r, s)$-gf $\delta$ so, $(r, s)$-gf $\delta$ po and $(r, s)$-gf $\beta o$ ) for any $\mu$ is $(r, s)$-gfeo (resp. $(r, s)-g f \delta s o$, $(r, s)-g f \delta p o$ and $(r, s)-g f \beta o)$.

(ii) $F^{u}(\mu)$ is $(r, s)$-gfec (resp. $(r, s)$-gf $\delta s c,(r, s)$-gf $\delta p c$ and $(r, s)-g f \beta c)$ for any $\mu$ is $(r, s)$-gfec (resp. $(r, s)-g f \delta s c$, $(r, s)-g f \delta p c$ and $(r, s)-g f \beta c)$.

Then $F$ is FLGe-irresolute.

Proof. This can be proved in similar way as Theorem 3.5 
Definition 3.8. A dfts $(X, \hat{\tau}, \breve{\tau})$ is said to be $(r, s)-F g e T_{\frac{1}{2}}$ (resp. $(r, s)-F g \delta s T_{\frac{1}{2}},(r, s)-F g \delta p T_{\frac{1}{2}}$ and $\left.(r, s)-F g \beta T_{\frac{1}{2}}\right)$ iff $\hat{\tau}(\underline{1}-\lambda) \geq$ $r, \breve{\tau}(\underline{1}-\lambda) \leq$ sfor each $\lambda \in L^{X}$ is $(r, s)$-gfec (resp. $(r, s)$-gf $\delta s c$, $(r, s)-g f \delta p c$ and $(r, s)-g f \beta c)$.

Example 3.9. Let $X=\left\{x_{1}, x_{2}\right\}, Y=\left\{y_{1}, y_{2}, y_{3}\right\}$ and $F: X \multimap$ $Y$ be a FM defined by $G_{F}\left(x_{1}, y_{1}\right)=0.2, G_{F}\left(x_{1}, y_{2}\right)=0.8$, $G_{F}\left(x_{1}, y_{3}\right)=0.3, G_{F}\left(x_{2}, y_{1}\right)=0.5, G_{F}\left(x_{2}, y_{2}\right)=0.3, G_{F}\left(x_{2}\right.$, $\left.y_{3}\right)=0.7$. Define double fuzzy topologies $\bar{\tau}, \breve{\tau}: L^{X} \rightarrow L$ and $\hat{\eta}, \breve{\eta}: L^{Y} \rightarrow$ L as follows:

$$
\begin{aligned}
& \hat{\tau}(\lambda)=\left\{\begin{array}{l}
1, \text { if } \lambda \in\{\underline{0}, \underline{1}\} \\
\frac{1}{2}, \text { if } \lambda=\underline{0.4} \\
0, \text { otherwise }
\end{array} \quad \breve{\tau}(\lambda)=\left\{\begin{array}{l}
0, \text { if } \lambda \in\{\underline{0}, \underline{1}\} \\
\frac{1}{2}, \text { if } \lambda=\underline{0.4} \\
1, \text { otherwise }
\end{array}\right.\right. \\
& \hat{\eta}(\mu)=\left\{\begin{array}{l}
1, \text { if } \lambda \in\{\underline{0}, \underline{1}\} \\
\frac{1}{2}, \text { if } \lambda=\underline{0.65} \\
0, \text { otherwise }
\end{array} \quad \breve{\eta}(\mu)=\left\{\begin{array}{l}
0, \text { if } \lambda \in\{\underline{0}, \underline{1}\} \\
\frac{1}{2}, \text { if } \lambda=\underline{0.65} \\
1, \text { otherwise }
\end{array}\right.\right.
\end{aligned}
$$

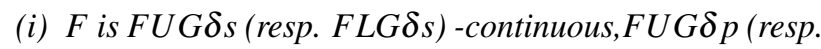
$F L G \delta p$ )-continuous but not FUG (resp. FLG)-continuous because $\hat{\eta}(0.65)=\frac{1}{2}=\breve{\eta}(0.65)$ in $(Y, \hat{\eta}, \breve{\eta})$ and $F^{u}(\underline{0.65})=\underline{0.65}\left(\operatorname{resp} . F^{l}(\underline{0.65})=\underline{0.65}\right)$ is not $\left(\frac{1}{2}, \frac{1}{2}\right)-g$ fo.

(ii) $F$ is $F U G \delta$ s (resp. FLG $\delta$ s)-irresolute, $F U G \delta p$ (resp. $F L G \delta p)$-irresolute but not FUG (resp. FUG)-irresolute because $\underline{0.65}$ is $\left(\frac{1}{2}, \frac{1}{2}\right)$-gfo in $(Y$, $\hat{\eta}, \breve{\eta})$ and $F^{u}(\underline{0.65})=F^{l}(\underline{0.65})=\underline{0.65}$ is not $\left(\frac{1}{2}, \frac{1}{2}\right)$ gfo.

Example 3.10. Let $X=\left\{x_{1}, x_{2}\right\}, Y=\left\{y_{1}, y_{2}, y_{3}\right\}$ and $F$ : $X \multimap Y$ be a FM defined by $G_{F}\left(x_{1}, y_{1}\right)=0.2, G_{F}\left(x_{1}, y_{2}\right)=$ $0.8, G_{F}\left(x_{1}, y_{3}\right)=0.3, G_{F}\left(x_{2}, y_{1}\right)=0.5, G_{F}\left(x_{2}, y_{2}\right)=0.3$, $G_{F}\left(x_{2}, y_{3}\right)=0.7$. Define double fuzzy topologies $\hat{\tau}, \breve{\tau}: L^{X} \rightarrow L$ and $\hat{\eta}, \breve{\eta}: L^{Y} \rightarrow$ L as follows:

$\hat{\tau}(\lambda)=\left\{\begin{array}{l}1, \text { if } \lambda \in\{\underline{0}, \underline{1}\} \\ \frac{1}{2}, \text { if } \lambda=\underline{0.65} \\ 0, \text { otherwise }\end{array} \quad \breve{\tau}(\lambda)=\left\{\begin{array}{l}0, \text { if } \lambda \in\{\underline{0}, \underline{1}\} \\ \frac{1}{2}, \text { if } \lambda=\underline{0.65} \\ 1, \text { otherwise }\end{array}\right.\right.$
$\hat{\eta}(\mu)=\left\{\begin{array}{l}1, \text { if } \lambda \in\{\underline{0}, \underline{1}\} \\ \frac{1}{2}, \text { if } \lambda=\underline{0.4} \\ 0, \text { otherwise }\end{array} \quad \breve{\eta}(\mu)=\left\{\begin{array}{l}0, \text { if } \lambda \in\{\underline{0}, \underline{1}\} \\ \frac{1}{2}, \text { if } \lambda=\underline{0.4} \\ 1, \text { otherwise }\end{array}\right.\right.$

(i) F is FUGe (resp. FLGe)-continuous, but not FUG $\delta$ s (resp. FLG $\delta$ s)-continuous because $\hat{\eta}(0.4)=\frac{1}{2}=$ $\breve{\eta}(0.4)$ in $(Y, \hat{\eta}, \breve{\eta})$ and $F^{u}(\underline{0.4})=\underline{0.4}\left(\right.$ resp. $F^{l}(\underline{0.4})=$ 0.4) is not $\left(\frac{1}{2}, \frac{1}{2}\right)-g f \delta$ so.

(ii) F is FUGe (resp. FLGe)-irresolute, but not FUG $\delta$ s (resp. $F U G \delta$ s)-irresolute because $\underline{0.4}$ is $\left(\frac{1}{2}, \frac{1}{2}\right)$ gf $\delta$ so in $(Y, \hat{\eta}, \breve{\eta})$ and $F^{u}(\underline{0.4})=F^{l}(\underline{0.4})=\underline{0.4}$ is not $\left(\frac{1}{2}, \frac{1}{2}\right)-g f \delta$ so.
Example 3.11. Let $X=\left\{x_{1}, x_{2}\right\}, Y=\left\{y_{1}, y_{2}, y_{3}\right\}$ and $F$ : $X \multimap Y$ be a FM defined by $G_{F}\left(x_{1}, y_{1}\right)=0.1, G_{F}\left(x_{1}, y_{2}\right)=$ $0.9, G_{F}\left(x_{1}, y_{3}\right)=0.3, G_{F}\left(x_{2}, y_{1}\right)=0.5, G_{F}\left(x_{2}, y_{2}\right)=0.3$, $G_{F}\left(x_{2}, y_{3}\right)=0.9$ Define double fuzzy topologies $\hat{\tau}, \breve{\tau}: L^{X} \rightarrow L$ and $\hat{\eta}, \breve{\eta}: L^{Y} \rightarrow$ L as follows:

$$
\begin{aligned}
& \hat{\tau}(\lambda)=\left\{\begin{array}{l}
1, \text { if } \lambda \in\{\underline{0}, \underline{1}\} \\
\frac{1}{2}, \text { if } \lambda=\{\underline{0.1}, \underline{0.6}\} \\
0, \text { otherwise }
\end{array} \quad \breve{\tau}(\lambda)=\left\{\begin{array}{l}
0, \text { if } \lambda \in\{\underline{0}, \underline{1}\} \\
\frac{1}{2}, \text { if } \lambda=\{\underline{0.1}, \underline{0.6}\} \\
1, \text { otherwise }
\end{array}\right.\right. \\
& \hat{\eta}(\mu)=\left\{\begin{array}{l}
1, \text { if } \lambda \in\{\underline{0}, \underline{1}\} \\
\frac{1}{2}, \text { if } \lambda=\underline{0.4} \\
0, \text { otherwise }
\end{array} \breve{\eta}(\mu)=\left\{\begin{array}{l}
0, \text { if } \lambda \in\{\underline{0}, \underline{1}\} \\
\frac{1}{2}, \text { if } \lambda=\underline{0.4} \\
1, \text { otherwise }
\end{array}\right.\right.
\end{aligned}
$$

(i) F is FUGe (resp. FLGe)-continuous, but not FUG $\delta$ p (resp. FLG $\delta$ p)-continuous because, $\hat{\eta}(0.4)=\frac{1}{2}=\breve{\eta}(0.4)$ in $(Y, \hat{\eta}, \breve{\eta})$ and $F^{u}(\underline{0.4})=\underline{0.4}$ (resp. $\left.F^{l}(\underline{0.4})=\underline{0.4}\right)$ is not $\left(\frac{1}{2}, \frac{1}{2}\right)-g f \delta$ po.

(ii) F is FUGe (resp. FLGe)-irresolute, but not FUG $\delta p$ (resp. $F U G \delta$ p)-irresolute because $\underline{0.4}$ is $\left(\frac{1}{2}, \frac{1}{2}\right)$-gf $\delta$ po in $(Y, \hat{\eta}, \breve{\eta})$ and $F^{u}(\underline{0.4})=F^{l}(\underline{0.4})=\underline{0.4}$ is not $\left(\frac{1}{2}, \frac{1}{2}\right)$ gf $\delta$ po.

Example 3.12. Let $X=\left\{x_{1}, x_{2}\right\}, Y=\left\{y_{1}, y_{2}, y_{3}\right\}$ and $F$ : $X \multimap Y$ be a $F M$ defined by $G_{F}\left(x_{1}, y_{1}=0.2, G_{F}\left(x_{1}, y_{2}=\right.\right.$ $0.8, G_{F}\left(x_{1}, y_{3}=0.3, G_{F}\left(x_{2}, y_{1}=0.5, G_{F}\left(x_{2}, y_{2}=0.3\right.\right.\right.$, $G_{F}\left(x_{2}, y_{3}=0.7\right.$. Define double fuzzy topologies $\tilde{\tau}, \breve{\tau}: L^{X} \rightarrow L$ and $\hat{\eta}, \breve{\eta}: L^{Y} \rightarrow$ L as follows:

$\hat{\tau}(\lambda)=\left\{\begin{array}{l}1, \text { if } \lambda \in\{\underline{0}, \underline{1}\} \\ \frac{1}{2}, \text { if } \lambda=\underline{0.8} \\ 0, \text { otherwise }\end{array} \quad \breve{\tau}(\lambda)=\left\{\begin{array}{l}0, \text { if } \lambda \in\{\underline{0}, \underline{1}\} \\ \frac{1}{2}, \text { if } \lambda=\underline{0.8} \\ 1, \text { otherwise }\end{array}\right.\right.$

$\hat{\eta}(\mu)=\left\{\begin{array}{l}1, \text { if } \lambda \in\{\underline{0}, \underline{1}\} \\ \frac{1}{2}, \text { if } \lambda=\underline{0.2} \\ 0, \text { otherwise }\end{array} \quad \breve{\eta}(\mu)=\left\{\begin{array}{l}0, \text { if } \lambda \in\{\underline{0}, \underline{1}\} \\ \frac{1}{2}, \text { if } \lambda=\underline{0.2} \\ 1, \text { otherwise }\end{array}\right.\right.$

(i) $F$ is FUG $\beta$ (resp. FLG $\beta$ )-continuous but not FUGe (resp. FLGe)-continuous because $\hat{\eta}(0.2)=\frac{1}{2}=$ $\breve{\eta}(0.2)$ in $(Y, \hat{\eta}, \breve{\eta})$ and $F^{u}(\underline{0.2})=\underline{0.2}\left(\operatorname{resp} . F^{u}(\underline{0.2})\right.$ $=\underline{0.2})$ is not $\left(\frac{1}{2}, \frac{1}{2}\right)$-gfeo.

(ii) F is FUG $\beta$ (resp. FLG $\beta$ )-irresolute but not FUGe (resp. FLGe)-irresolute because $\underline{0.2}$ is $\left(\frac{1}{2}, \frac{1}{2}\right)$-gfeo in $(Y, \hat{\eta}, \breve{\eta})$ and $F^{u}(\underline{0.2})=F^{l}(\underline{0.2})=\underline{0.2}$ is not $\left(\frac{1}{2}, \frac{1}{2}\right)$ gfeo.

Corollary 3.13. Let $F: X \multimap Y$ be a FM between two dfts's $(X, \hat{\tau}, \breve{\tau}),(Y, \hat{\eta}, \breve{\eta})$. The following statements hold.

(i) If $(X, \hat{\tau}, \breve{\tau})$ is $(r, s)-F g e T_{\frac{1}{2}}$ (resp. $F g \delta s T_{\frac{1}{2}}, F g \delta p T_{\frac{1}{2}}$ and $\left.F g \beta T_{\frac{1}{2}}\right)$ then the concepts of FUGe (resp. FUG $\delta$, FUG $\delta$ p and FUG $\beta$ )-continuous are equivalent. Also the concepts of FLGe (resp. FLG $\delta$ s, FLG $\delta$ p and FLG $\beta$ )continuous are equivalent. 
(ii) If $(Y, \hat{\eta}, \breve{\eta})$ is $(r, s)-F g e T_{\frac{1}{2}}$ (resp. $\quad F g \delta s T_{\frac{1}{2}}, F g \delta p T_{\frac{1}{2}}$ and $F g \beta T_{\frac{1}{2}}$ ) then the concepts of FUGe (resp. FUG $\delta s, F U G \delta p$ and $F U G \beta$ )-continuous and FUGe (resp. $F U G \delta s, F U G \delta p$ and $F U G \beta$ )-irresolute are equivalent.

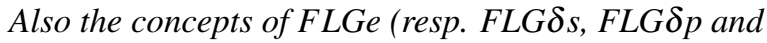
FLG $\beta$ )-continuous and FLGe (resp. FLG $\delta$ s, FLG $\delta p$ and FLG $\beta$ )-irresolute are equivalent.

(iii) If $(X, \hat{\tau}, \breve{\tau})$ and $(Y, \hat{\eta}, \breve{\eta})$ are $(r, s)-F g e T_{\frac{1}{2}}$ (resp. $F g \delta s T_{\frac{1}{2}}$, $F g \delta p T_{\frac{1}{2}}$ and $F g \beta T_{\frac{1}{2}}$ ) then the concepts of $F U G e$ (resp. $F U G \delta s, F U G \delta p$ and FUG $\beta$ )-continuous, FUGe (resp. $F U G \delta s, F U G \delta p$ and $F U G \beta$ )-irresolute are equivalent. Also the concepts of FLGe (resp. FLG $\delta$ s, FLG $\delta$ p and

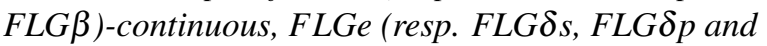
$F L G \beta$ )-irresolute are equivalent.

Remark 3.14. From the above examples it is clear that the following implications are true:

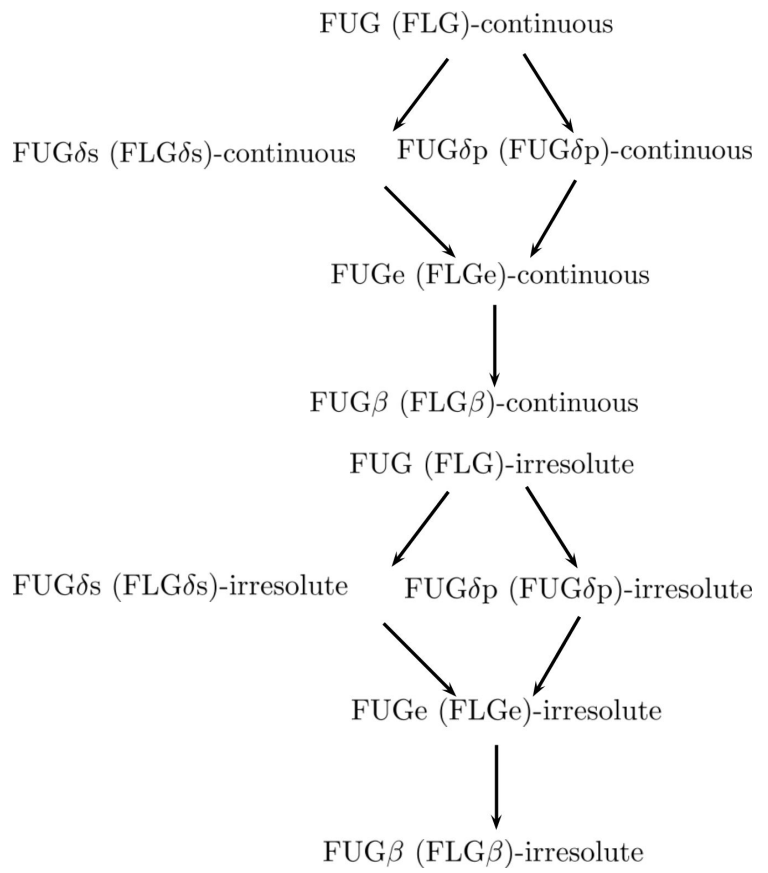

In general the converse are not true as shown in above examples.

\section{References}

[1] S. E. Abbas, $(r, s)$-generalized intuitionistic fuzzy closed sets, J. Egypt Math. Soc., 14(2)(2006), 283-297.

[2] S. E. Abbas and E. El-Sanousy, Several types of double fuzzy semiclosed sets, J. Fuzzy Math., 20(1)(2012), 89102.

[3] S. E. Abbas, M. A. Hebeshi and I. M. Taha, Continuity for fuzzy multifunctions (submitted for publication).

[4] K. M. A. Al-hamadi and S. B. Nimse, On fuzzy $\alpha$ continuous multifunctions, Miskolc Mathematical Notes, 11(2)(2010), 105-112.
[5] M. Alimohammady, E. Ekici, S. Jafari and M. Roohi On fuzzy upper and lower continuous multifunctions, Iranian Journal of Fuzzy Systems, 8(3)(2011), 149-158.

[6] K. Atanassov, Intuitionistic fuzzy sets, Fuzzy Sets and Systems, 20(1984), 87-90.

[7] K. Atanassov, New operators defined over the intuitionistic fuzzy sets, Fuzzy Sets and Systems, 61(2)(1993), 131-142.

[8] K. Atanassov and S. Stoeva, Intuitionistic fuzzy sets, In Proceedings of Polish Symposium on Interval and Fuzzy Mathematics, (1983), 23-26.

[9] C. Berge, Topological spaces, Including a treatment of Multi-Valued functions, Vector spaces and convexity, Oliver, Boyd London, (1963).

[10] C. L. Chang, Fuzzy topological spaces,Journal of Mathematical Analysis and Applications, 24(1968), 182-190.

[11] D. Coker, An introduction to fuzzy subspaces in intuitionistic fuzzy topological spaces, Journal of Fuzzy Mathematics, 4(1996), 749-764.

[12] D. Coker, An introduction to intuitionistic fuzzy topological spaces, Fuzzy Sets and Systems, 88(1997), 81-89.

[13] D. Coker and M. Demirci, An introduction to intuitionistic fuzzy topological spaces in Sostaks sense, BUSEFAL, 67(1996), 67-76.

[14] J. G. Garcia and S. E. Rodabaugh, Order-theoretic, topological, categorial redundancies of interval-valued sets, grey sets, vague sets, intervalued "intuitionistic" sets, "intuitionistic" fuzzy sets and topologies, Fuzzy Sets and Systems, 156(2005), 445-484.

[15] J. A. Goguen, The fuzzy tychonoff theorem, Journal of Mathematical Analysis and Applications, 43(1973), 734742.

[16] M. A. Hebeshi and I. M. Taha, On Double Fuzzy Topological Spaces, The Journal of Fuzzy Mathematics, 22(2)(2014), 419-434.

[17] U. Hohle, Upper semicontinuous fuzzy sets and applications, Journal of Mathematical Analysis and Applications 78(1980), 659-673.

[18] U. Hohle and A. P. Sostak, A general theory of fuzzy topological spaces, Fuzzy Sets and Systems, 73(1995), 131-149.

[19] U. Hohle and A. P. Sostak, Axiomatic foundations of fixedbasis fuzzy topology, The Hand-books of fuzzy sets series, 3, Kluwer academic publishers, Dordrecht (Chapter 3) (1999).

[20] T. Kubiak, On fuzzy topologies, Ph.D. Thesis, A. Mickiewicz, Poznan (1985).

[21] T. Kubiak and A. P. Sostak, Lower set-valued fuzzy topologies, Questions Math., 20(3)(1997), 423-429.

[22] E. P. Lee and Y. B. Im, Mated fuzzy topological spaces, Journal of Fuzzy Logic and Intelligent Systems, (Korea), 11(2)(2001), 161-165.

[23] E. P. Lee, Semiopen sets on intuitionistic fuzzy topological spaces in Sostak's sense, Journal of Fuzzy Logic and Intelligent Systems, (Korea), 14(2004), 234-238. 
[24] S. L. Lee and E.P. Lee, Fuzzy $(r, s)$-semicontinuous mappings on intuitionistic fuzzy topological spaces Ŝstak's sense, Journal Of fuzzy logic and intelligent systems (Korea), 16(2006), 108-112.

[25] R. A Mahmoud, An application of continuous fuzzy multifunctions, Chaos, Solitons and Fractals, 17 (2003), 833841.

[26] M. N. Mukherjee and S. Malakar, On almost continuous and weakly continuous fuzzy multifunctions, Fuzzy Sets and Systems, 41(1991), 113-125.

[27] N. S. Papageorgiou, Fuzzy topology an fuzzy multifunctions, Journal of Mathematical Analysis and Applications, 109(1985), 397-425.

[28] P. Periyasamy, V. Chandrasekar, G. Saravanakumar and A. Vadivel, Fuzzy e-closed and Generalized fuzzy e-closed sets in Double fuzzy topological spaces. (submitted)

[29] V. Popa, On characterizations of irresolute multimapping, Journal of University of Kuwait(Sci)., 15(1988),21-25.

[30] V. Popa, Irresolute multifunctions, Internat. Journal of Mathematical Analysis and Applications, 13(2)(1990), 275-280.

[31] S. K. Samanta and T. K Mondal, Intuitionistic gradation of opennes, Intuitionistic Fuzzy Topology, Busefal, 73(1997), 8-17.

[32] S. K. Samanta and T. K Mondal, On Intuitionistic gradation of opennes, Fuzzy Sets and Systems, 131(2002), 323-336.

[33] A. P. Ŝstak, On a fuzzy topological structure, Suppl. Rend. Circ. Matem. Palerms, Ser II 11 (1985), 89-103.

${ }^{[34]}$ E. Tsiporkova, B. De Baets and E. Kerre, A fuzzy inclusion based approach to upper inverse images under fuzzy multivalued mappings, Fuzzy Sets and System, 85(1997), 93-108.

[35] E. Tsiporkova, B. De Baets and E. Kerre, Continuity of fuzzy multivalued mappings, Fuzzy Sets and System, 94(1998), 335-348.

[36] A. M. Zahran, M.A. Abd-Allah and A. Ghareeb, Several types of double fuzzy irresolute functions, International Journal of Computational Cognition 8(2)(2010), 19-23.

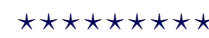

$\operatorname{ISSN}(\mathrm{P}): 2319-3786$

Malaya Journal of Matematik

ISSN(O):2321-5666

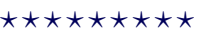

